\title{
Competencias de desempeño mediadas por las TIC para el fortalecimiento de la calidad educativa. Una revisión sistemática
}

\section{Performance competencies mediated by ICT for the strengthening of educational quality. A systematic review}

DOI: http://dx.doi.org/10.17981/cultedusoc.10.1.2019.07

Recibido: Agosto 02 de 2018 Aceptado: Abril 26 de 2019

\author{
Carolina Mercado-Porras \\ Universidad de la Costa (Colombia) \\ cmercado6@cuc.edu.co
}

Yuneidis Morales-Ortega

Universidad de la Costa (Colombia) ymorales4@cuc.edu.co

Para citar este artículo:

Mercado-Porras, C. y Morales-Ortega, Y. (2019). Competencias de desempeño mediadas por las TIC para el fortalecimiento de la calidad educativa. Una revisión sistemática. Cultura, Educación y Sociedad, 10(1). 109-124. DOI: http://dx.doi.org/10.17981/ cultedusoc.10.1.2019.07

\section{Resumen}

El mundo de las Tecnologías de la Información y la Comunicación (TIC) ha revolucionado el escenario educativo, planteando nuevas alternativas para el desarrollo de las capacidades de los profesionales en los diversos contextos. El presente artículo tiene el propósito de analizar las competencias de desempeño implementadas bajo el uso de las TIC, como un factor de impacto para el fortalecimiento de la calidad educativa en las Instituciones de Educación Superior (IES). El abordaje metodológico ha comprendido el desarrollo de una investigación documental con enfoque cualitativo, con una técnica instrumental para la revisión sistemática de (50) artículos especializados publicados en revistas de alto impacto (Scopus - WoS). Los resultados permiten aproximarse al estado del arte desde un sistema teórico, identificando las principales implementaciones de las TIC en el ámbito educativo, logrando evidenciar los lineamientos estratégicos que fortalecen el proceso de desarrollo de competencias de desempeño de acuerdo con las etapas de formación definidas en las IES.

Palabras clave: Tecnologías de información y comunicación (TIC); calidad de la enseñanza, competencia de desempeño; educación superior; enseñanza y formación

\begin{abstract}
The world of Information and Communication Technologies (ICT) has revolutionized the educational scenario, proposing new alternatives for the development of the capacities of professionals in different contexts. The purpose of this article is to analyze the performance competencies implemented under the use of ICT, as an impact factor for the strengthening of educational quality in Higher Education Institutions (HEI). The methodological approach has included the development of a documentary research with a qualitative focus, with an instrumental technique for the systematic review of (50) specialized articles published in high-impact journals (Scopus - WoS). The results make it possible to approach the state of the art from a theoretical system, identifying the main implementations of ICT in the educational field, making evident the strategic guidelines that strengthen the process of development of performance competencies in accordance with the stages of training defined in the HEIs.
\end{abstract}

Keywords: Information and communication technologies (ICTs); quality of teaching; performance competence; higher education; education and training 


\section{INTRODUCCIÓN}

A partir de la última década, en el país se ha evidenciado como las Instituciones de Educación Superior (IES) han afrontado los retos para abordar los diferentes aspectos de una educación pertinente y diferenciadora que permita la generación de una alta calidad en los procesos educativos y la inclusión de nuevos saberes, habilidades, herramientas y competencias que aporten al desarrollo de la enseñanza y el aprendizaje.

Como lo establecen las Ley 115 (1994) y la Ley 30 (1992), la educación en Colombia se define como un proceso de formación permanente, personal, cultural y social que se fundamenta en una concepción integral de la persona humana, de su dignidad, sus derechos y sus deberes. En este sentido, la educación es un valor esencial para todos los ciudadanos, en donde el Estado en conjunto con las diferentes instituciones u organismos reguladores, adoptan medidas para garantizar el acceso al sistema de formación; a través del monitoreo y control constante enfocados a la generación de su alta calidad, es así como el Ministerio de Educación Nacional en Colombia (MEN) ha constituido proyectos que puedan desarrollar, asegurar y transformar ésa calidad educativa en los diferentes niveles de formación Nacional.

En éste contexto referencial se puede inferir que el logro del objetivo antes mencionado, parte de la identificación de estrategias que permitan fortalecer el aprendizaje desarrollado por los profesionales en las Instituciones de Educación Superior. Entre las estrategias más significativas se encuentra las referidas a la integración de Tecnologías de Información y Comunicación (TIC), como una estrategia de mediación para el fortalecimiento didáctico.

La relación de las referidas estrategias se ha venido consolidando en el marco de significativas transformaciones de la edu- cación, que orientan el desarrollo de modelos de aprendizaje basados en la formación por competencias. Desde esta perspectiva la implementación de nuevas tecnologías impulsa procesos de enseñanza y aprendizaje que inciden en la formación de profesionales integrales con capacidades para participar en la solución de diferentes problemas y procesos propios de un mundo globalizado.

Con base en los anteriores planteamientos, las Instituciones de Educación Superior avaladas por el Ministerio de Educación en Colombia han focalizado su quehacer a la integración de las TIC en el aula. Para ello, la UNESCO (Organización de las Naciones Unidas para la Educación, la Ciencia y la Cultura) ha estipulado unos estándares de competencias en TIC para los docentes, a fin de proporcionar un conjunto de competencias y conocimientos que deben ser adquiridos por lo docentes para el desarrollo de estrategias y prácticas eficaces, que permitan aprovechar las nuevas posibilidades educativas en tecnologías, contribuyendo a una educación para todos (UNESCO, 2008). De forma tal, que se deba tener la disposición para afrontar éste ejercicio, con la capacidad que las personas participen activamente del aprendizaje tecnológico.

Karam, Buitrago, Fagua y Romero (2013) destacan la importancia de las Instituciones educativas al incorporar las TIC en su plan de trabajo, para lo cual sugieren varias etapas: 1) Sistematización de los sistemas administrativos, 2) Dotación de aulas de informática, 3) Sistematización de los sistemas académicos, 4) Uso de TIC en clases presenciales diferentes a informática y, 5) Uso de aulas virtuales y espacios WEB. En este contexto, las TIC se ha convertido en un factor diferenciador entre las Instituciones Educativas. La implementación requiere redefinir un nuevo sistema educativo que contribuya al fortalecimiento del perfil por competencia en estudiantes y docentes. 
Desde los lineamientos expuestos por el Ministerio de Educación Nacional (2013), las competencias para el desarrollo de la innovación educativa apoyadas por las TIC son: tecnológica, comunicativa, pedagógica, investigativa y de gestión; las referidas competencias resultan de importancia fundamental para afianzar las estrategias didácticas-pedagógicas, que permitan la gestión curricular por cuanto se consideran las particularidades propias al proceso educativo y los cambios del contexto de forma tal, que se vayan fortaleciendo el perfil personal y profesional de los actores involucrados. Para lograr una comprensión amplia del concepto de competencias en el ámbito de las Tecnologías de la Información y la Comunicación, es necesario analizar la concepción de competencia en un concepto más genérico.

En un sentido genérico, diversos han sido los aportes para comprender las concepciones acerca de la definición de competencias. En tal sentido, autores como Tobón (2006) y Marín-González, Cabas, Cabas y Paredes-Chacín (2018) sustentan como el enfoque centrado en competencias es fundamentado por la integración de conocimientos, procesos cognitivos, destrezas, habilidades, valores, actitudes en el desempeño; considerados también como los requerimientos de las disciplinas, profesiones y contextos, todo ello en correspondencia con los estándares de calidad, a su vez, identifica competencias genéricas comunes con disciplinas o profesiones y las específicas que dan identidad a cada ocupación, implicando ambas como perfiles de desempeño.

Los programas académicos universitarios deben fortalecer el desempeño por competencias asociados a la gestión curricular, resulta importante consolidar una educación integral que permita la inclusión de metodologías innovadoras y pertinentes donde se evalúe la consistencia del currículo y se propicie el mejoramiento continuo de la calidad todo ello en el marco de los procesos de enseñanza y aprendizaje y desarrollo de los perfiles profesionales.

En el presente artículo, se analiza el desarrollo de las competencias de desempeño mediadas por las TIC que orientan la tarea pedagógica y trazan las perspectivas del futuro de la educación. A raíz de la revisión documental, se logrará minimizar las brechas que existe entre algunas Instituciones para la implementación de metodologías basadas en TIC aplicadas a su Proyecto Educativo Institucional (PEI) como factor diferenciador que apunta al mejoramiento continuo para el logro de la calidad educativa.

Al respecto Sunkel, Trucco y Espejo (2014), Pinto y Díaz (2015), Nolasco y Ojeda (2016) y Marín, Inciarte, Hernández y Pitre (2017) resaltan la necesidad de que los sistemas educativos disminuyan la brecha en el acceso al mundo digital como alternativa válida para el fortalecimiento del desempeño académico, formación docente, cobertura y calidad educativa. Analizando cómo la implementación de las TIC aplicadas a los currículos académicos, puede influenciar específicamente al desarrollo de competencias de desempeño en la Educación Superior.

\section{Metodología}

La investigación asume un diseño metodológico de indagación documental, sustentada en el proceso de revisión sistemática de artículos de investigación publicados en revistas de alto impacto (Scopus / WoS). La principal técnica abordada se fundamentó en la revisión sistemática, considerando los aportes realizados por Moher, Liberati, Tetzlaff, Altman \& The Prisma Group (2009), donde se logra ubicar la información clave en un vínculo seleccionado de fuentes; este referente conlleva a discriminar las de mayor pertinencia que corresponden a un espacio teórico-conceptual establecido en diferentes categorías trabajadas. 
Una vez procesada la información se procede a un análisis cualitativo, identificando en las bases de datos de consultas especializadas un conjunto de 50 artículos seleccionados, acorde a la temática de investigación y a partir de los criterios de inclusión correspondientes a su publicación en Revistas indexadas de alto impacto (Scopus/ WoS), disponibles en texto completo dentro de la Base de ScienceDirect; en relación con la naturaleza del trabajo seleccionado se toma en consideración grado de congruencia en la temática e indicadores de calidad.

El procesamiento de la información obtenida de las bases de datos, se trabaja mediante la abstracción de conceptos de los diferentes autores identificados en las fuentes en relación con las categorías trabajadas. El análisis se sustenta en los componentes más significativos que estructuran a cada artículo en revisión y que son fuente base para la elaboración y distribución de matrices de análisis y revisión.
En síntesis, la investigación fue desarrollada bajo una revisión sistemática que implicó la siguiente ruta de etapas: análisis estratégico y prospectivo de la temática, delimitación del eje temático, elaboración del plan de trabajo, consulta especializada de información, análisis de artículos de alto impacto, elaboración de la matriz de revisión sistemática, proceso de incorporación referencial, redacción del artículo y revisión de criterios de calidad científica y editorial.

\section{Resultados}

En el presente apartado, se resaltan los principales hallazgos derivados de la revisión sistemática adelantada frente al sistema educativo con la implementación de las TIC en el desarrollo de competencias de desempeño. Estructurados en las siguientes tablas analíticas presentadas a continuación:

TABLA 1

Revisión Sistemática de Estudios sobre la Calidad Educativa en la Educación Superior

\begin{tabular}{|c|c|c|c|c|}
\hline Año & Autores & Título & $\begin{array}{c}\text { Categoría Central } \\
\text { de Análisis }\end{array}$ & Postura Argumentativa \\
\hline 2018 & $\begin{array}{l}\text { Freddy } \\
\text { Marín- } \\
\text { González, } \\
\text { Lorena de } \\
\text { J. Cabas, } \\
\text { Luis C. } \\
\text { Cabas y } \\
\text { Ana J. } \\
\text { Paredes- } \\
\text { Chacín }\end{array}$ & $\begin{array}{l}\text { Formación integral } \\
\text { en profesionales } \\
\text { de la ingeniería. } \\
\text { análisis en el } \\
\text { plano de la calidad } \\
\text { educativa }\end{array}$ & $\begin{array}{l}\text { Competencia } \\
\text { Calidad Educativa } \\
\text { Formación Integral }\end{array}$ & $\begin{array}{l}\text { Con las diferentes concepciones para la } \\
\text { formación integral de los profesionales } \\
\text { correspondiente al programa académico } \\
\text { de ingeniería en Colombia, se propende } \\
\text { realizar espacios culturales que } \\
\text { permitan identificar y abordar las } \\
\text { necesidades comunes, transferencias e } \\
\text { intercambios de conocimientos básicos } \\
\text { aplicados, de una manera clave para el } \\
\text { desarrollo de individuos competentes, } \\
\text { emprendedores y capaces de adaptarse } \\
\text { al entorno. Logrando así concebir en las } \\
\text { Instituciones de Educación Superior el } \\
\text { desarrollo de competencias integrales, } \\
\text { articuladas a las dimensiones } \\
\text { axiológicas, socioafectivas, cognitivas } \\
\text { y actitudinales en consideración de los } \\
\text { estándares de calidad. }\end{array}$ \\
\hline
\end{tabular}




Año Autores $\quad$ Título $\quad \begin{gathered}\text { Categoría Central } \\ \text { de Análisis }\end{gathered}$

Postura Argumentativa

Los grandes retos y desafíos en las reformas de modernización institucional que enfrentan los modelos de calidad educativa superior en países como México, han permitido ampliar la cobertura de los programas de pregrado, aliviando las presiones financieras del sector educativo. Las diferentes investigaciones realizadas en torno

Las reformas

Carlos Iván en la educación

2017 Moreno superior pública en

Arellano México: rupturas y continuidades
Calidad Educativa

Educación Superior
Calidad del

Servicio

Sistemas

Educativos a éste fenómeno han evidenciado que algunos centros de educación superior en este país, le dan la bienvenida a los cambios de las políticas públicas, las cuales son aprovechadas para lograr el avance en la educación superior, pues así, se genera oportunidades para la generación de nuevos programas institucionales con una alta calidad, introduciendo valiosos cambios en los mecanismos de gobierno, gestión y administración, que contribuyan al desarrollo de la innovación y la competencia internacional.

En toda Institución Educativa es indispensable contar con diversas dimensiones que aportan significativamente a la calidad de servicio en los sistemas educativos, tales como la cualificación de la planta docente, las instalaciones físicas de las instituciones, el desarrollo integral del estudiante en función a su formación humana y la combinación de aspectos teóricos-metodológicos que respaldan las diferentes maneras de medir la calidad. No cabe dudas, que con el estudio realizado se logra identificar el nivel de percepción de los estudiantes del Instituto Tecnológico de Estudios Superiores de Monterrey y la Universidad Autónoma de Nuevo León en cuanto a la calidad del servicio ofrecido, considerando las dimensiones antes indicadas. Para algunos alumnos les dan mayor peso a las instalaciones con avances tecnológicos y modernos, mientras otros valoran más la preparación académica que tienen los profesores. 


Año Autores $\quad$ Título $\quad \begin{gathered}\text { Categoría Central } \\ \text { de Análisis }\end{gathered} \quad$ Postura Argumentativa

En la transición entre los años 80 y 90 se percibía una necesidad de cambio que permitiera la generación de una nueva mentalidad en el contexto de las políticas de evaluación y acreditación universitaria. Es precisamente en esos periodos donde se concebía la calidad como una variable multifacética, a tal punto que algunas de las perspectivas del poder ejercidas por las delegaciones de pares evaluadores en el contexto educativo de los Institutos Universitarios en Latinoamérica, permitiera analizarse los modelos de de la educación pública y evaluar la Calidad $2014 \quad \begin{aligned} & \text { Marcela } \\ & \text { Mollis }\end{aligned}$ calidad universitaria Institucional en América Latina: Evaluación y dos caras de la Acreditación las reformas en las acreditaciones, y títulos universitarios que aporten significativamente a la calidad de profesores, estudiantes, programas e infraestructura institucional; misma reforma educativa encontrando con ello soluciones a los posibles problemas o crisis presentados en el sistema educativo. El reformar el legado de las universidades logrará reconocer y evidenciar las necesidades y debilidades que ésta pueda presentar, es allí como el autor propende la existencia de una reconstrucción en la misión institucional que pueda involucrar la trilogía: aprender para innovar; enseñar para recrear e investigar para producir ciencia, tecnología y cultura.

Al considerarse los análisis realizados sobre los datos oficiales del sistema de aseguramiento de la calidad en la Educación Superior de Chile, se logra evidenciar algunas inconsistencias entre el gobierno y las instituciones en relación a los procesos de calidad, teniendo claro que las universidades se encuentran frente a una avalancha de retos que conllevan a la exigencia de

Oscar del sistema de

Espinoza aseguramiento de la

2012 y Luis

Eduardo González calidad y el régimen de acreditación en la educación superior en Chile
Aseguramiento de la calidad Acreditación futuros profesionales. Entre tanto, en cada país el Estado debería tomar un rol protagónico para la supervisión y regulación del sistema, especialmente para el cumplimiento de los criterios y estándares, asegurando la equidad para todos los beneficiarios del sistema de educación superior. Para los autores es muy importante que se instauren indicadores, que puedan evidenciarse los avances de las políticas, estrategias $\mathrm{y}$ acciones en el contexto nacional y regional. 


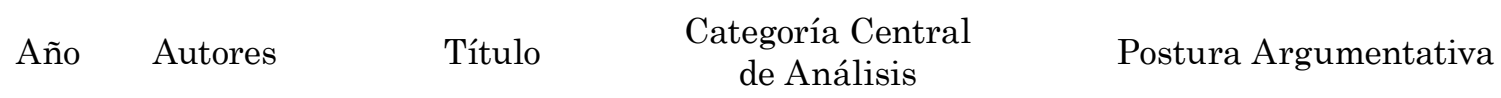

Las Instituciones de Educación Superior (IES) en relación a su servicio educativo enfrenta continuos cambios y consecuencias en el ámbito académico, proveniente a los efectos de la globalización en relación a la calidad avanzada en el sector de la educación, los diferentes requerimientos en la formación de tercer nivel, y los actuales modelos en el mercado laboral.

Instituciones de Educación Superior Calidad del Servicio Educativo Responsabilidad Social

\section{Visión}

Philip G.

Altbach,

2006 Jane

Knight panorámica de la Educación

internacionalización transfronteriza en la educación superior: motivaciones y realidades Aseguramiento de la calidad y acreditación
Es por ello, que se hace indispensable tener pertinencia en la calidad del servicio educativo brindado por las IES, los cuales deben considerar e instaurar nuevas estrategias y sistemas que puedan implementarse en los procesos organizacionales, de gestión, bienestar estudiantil y profesoral, poniendo mayor interés a la responsabilidad social de la institución y de los programas académicos; de allí que el autor afirma que no se puede seguir siendo instituciones exitosas en sociedades fracasadas.

El aporte realizado por los autores logra evidenciar aspectos como el desarrollo curricular, la integración regional, la generación de ingresos, entre otros; han marcado significativamente los cambios presentados en la Educación Superior Internacional. Argumentan y sustentan también, la importancia que le representa a las IES en torno al crecimiento de la educación transfronteriza, esencialmente para los fines de intercambio comercial; tomando con mayor vigor los resultados correspondientes al registro de proveedores extranjeros, aseguramiento de la calidad y acreditación de programas. Proporcionándole a la educación superior internacional un rol cada vez más importante en cuanto a la provisión de acceso y conformación de nuevos nichos del mercado por explorar. Si bien, una de las preocupaciones de los autores es el aseguramiento de la educación implementada para el bien público y no como un sistema para conseguir ganancias. 


\begin{tabular}{|c|c|c|c|c|}
\hline Año & Autores & Título & $\begin{array}{c}\text { Categoría Central } \\
\text { de Análisis }\end{array}$ & Postura Argumentativa \\
\hline 2004 & $\begin{array}{l}\text { Sara } \\
\text { Fernández } \\
\text { López y } \\
\text { Emilio } \\
\text { Ruzo } \\
\text { Sanmartín }\end{array}$ & $\begin{array}{l}\text { Los Procesos de } \\
\text { Internacionalización } \\
\text { y Globalización en la } \\
\text { Educación Superior: } \\
\text { Un Análisis de los } \\
\text { Países OCDE }\end{array}$ & $\begin{array}{l}\text { Calidad Educativa } \\
\text { Inter- } \\
\text { nacionalización } \\
\text { Servicios } \\
\text { educativos }\end{array}$ & $\begin{array}{l}\text { Los métodos de internacionalización } \\
\text { en la calidad educativa avanzada } \\
\text { son cada vez más importantes en } \\
\text { las políticas públicas de cada país, } \\
\text { los gestores involucrados, en la } \\
\text { educación mantienen un vínculo } \\
\text { representativo, que ratifica los servicios } \\
\text { educativos (exportables), ocasionando } \\
\text { nuevos conceptos o acciones que son } \\
\text { determinantes para la generación de } \\
\text { la regionalización, la cual promueve } \\
\text { el vencimiento de barreras u } \\
\text { obstáculos que den lugar al proceso de } \\
\text { internacionalización y globalización de } \\
\text { la educación superior, asegurando el } \\
\text { ingreso de más jóvenes a las IES con } \\
\text { una educación de la más alta calidad. }\end{array}$ \\
\hline
\end{tabular}

Fuente: Elaboración de los autores.

Es evidente observar como en un mundo tan cambiante y globalizado las Instituciones de Educación Superior (IES) se enfrentan a diversas condiciones tanto académicas como administrativas, reflejadas en la misión, imagen $\mathrm{y}$ documentos institucionales como el Proyecto Educativo Institucional (PEI), los cuales están llamados a cumplir. Por su parte, el gobierno es responsable de estipular dentro de su facultad los diferentes lineamientos y normas en pro al aseguramiento de la calidad educativa, como vía responsable para el avance o retraso de la educación; convirtiendo las IES en los garantes de llevar una buena gestión en coherencia de las políticas públicas.
Sin embargo, los efectos de la globalización y los escenarios cambiantes en el servicio, ponen a prueba la excelencia académica y la calidad educativa, convirtiéndose así en metas que todas las universidades a nivel nacional e internacional quieren lograr obtener. Para tal fin, deben implementarse estrategias innovadoras enmarcadas en el PEI, logrando con ello alcanzar los estándares establecidos para la obtención de su reconocimiento.

A continuación, se presenta los hallazgos más representativos sobre el uso de las TIC en las Instituciones de Educación Superior como herramienta encaminada al óptimo desarrollo y mejoramiento de la educación, integrando factores tecnológicos que acompañen a la generación del conocimiento y la innovación. 
TABLA 2

Análisis sobre la implementación de las TIC en la Educación Superior

\begin{tabular}{|c|c|c|c|c|}
\hline Año & Autores & Título & $\begin{array}{c}\text { Categoría Central } \\
\text { de Análisis }\end{array}$ & Postura Argumentativa \\
\hline 2017 & $\begin{array}{l}\text { Freddy V } \\
\text { Marín, Alicia } \\
\text { de J Inciarte, } \\
\text { Hugo G } \\
\text { Hernández, } \\
\text { Remedios C } \\
\text { Pitre }\end{array}$ & $\begin{array}{l}\text { Estrategias de } \\
\text { las Instituciones } \\
\text { de Educación } \\
\text { Superior para la } \\
\text { Integración de } \\
\text { las Tecnología de } \\
\text { la Información y } \\
\text { la Comunicación } \\
\text { y de la } \\
\text { Innovación en } \\
\text { los Procesos } \\
\text { de Enseñanza. } \\
\text { Un Estudio en } \\
\text { el Distrito de } \\
\text { Barranquilla, } \\
\text { Colombia }\end{array}$ & $\begin{array}{l}\text { Educación Superior } \\
\text { Enseñanza y } \\
\text { Aprendizaje } \\
\text { Tecnología }\end{array}$ & $\begin{array}{l}\text { En el estudio realizado por los autores } \\
\text { sobre el nivel de interacción que tienen } \\
\text { los docentes de las Instituciones de } \\
\text { Educación Superior con la tecnología, } \\
\text { se logró evidenciar que gran parte } \\
\text { de las instituciones analizadas en la } \\
\text { investigación, no proporcionan a los } \\
\text { docentes escenarios en donde puedan } \\
\text { capacitarse sobre la implementación } \\
\text { de las Tecnologías de la Información } \\
\text { y la Comunicación en el aula de } \\
\text { clase; adicional a ello, no desarrollan } \\
\text { metodologías innovadoras para } \\
\text { la enseñanza y aprendizaje que } \\
\text { propendan a fortalecer la didácticas } \\
\text { implementadas, logrando así incentivar } \\
\text { la participación de los estudiantes } \\
\text { durante su etapa formativa. }\end{array}$ \\
\hline 2017 & $\begin{array}{l}\text { Olga Gloria } \\
\text { Barbón } \\
\text { Pérez y Jorge } \\
\text { Washington } \\
\text { Fernández } \\
\text { Pino }\end{array}$ & $\begin{array}{l}\text { Rol de la gestión } \\
\text { educativa } \\
\text { estratégica en } \\
\text { la gestión del } \\
\text { conocimiento, } \\
\text { la ciencia, la } \\
\text { tecnología y la } \\
\text { innovación en } \\
\text { la educación } \\
\text { superior }\end{array}$ & $\begin{array}{l}\text { Aprendizaje } \\
\text { Innovación } \\
\text { TIC }\end{array}$ & $\begin{array}{l}\text { Las Universidades Ecuatorianas en } \\
\text { su afán por obtener cada día más } \\
\text { fuerza, requiere un perfeccionamiento } \\
\text { constante, en donde pueda asumir las } \\
\text { nuevas exigencias de la generación } \\
\text { de conocimientos, liderazgo científico } \\
\text { y de nuevas oportunidades que } \\
\text { permitan aplicar soluciones a } \\
\text { problemas referentes con la adquisición } \\
\text { del aprendizaje, la comunicación, } \\
\text { enseñanza, investigación y la } \\
\text { transferencia de datos, satisfaciendo así } \\
\text { las necesidades de cambio del entorno. }\end{array}$ \\
\hline 2013 & $\begin{array}{l}\text { Norma } \\
\text { Pedraza, } \\
\text { Gabriela } \\
\text { Farías, Jesús } \\
\text { Lavín y Aldo } \\
\text { Torres }\end{array}$ & $\begin{array}{l}\text { Las } \\
\text { competencias } \\
\text { docentes en TIC } \\
\text { en las áreas } \\
\text { de negocios } \\
\text { y contaduría } \\
\text { Un estudio } \\
\text { exploratorio en } \\
\text { la educación } \\
\text { superior }\end{array}$ & $\begin{array}{l}\text { Recursos } \\
\text { Tecnológicos } \\
\text { Competencias } \\
\text { Pedagógicas } \\
\text { Ambientes } \\
\text { Educativos }\end{array}$ & $\begin{array}{l}\text { La visión particular de los docentes } \\
\text { de educación superior en México, } \\
\text { permiten comprender la necesidad de } \\
\text { que se incrementen los programas de } \\
\text { capacitación y desarrollo permanentes } \\
\text { para los docentes, que les provean } \\
\text { entrenamiento para el aumento } \\
\text { de habilidades que les permitan } \\
\text { incorporar estos recursos tecnológicos } \\
\text { (competencias pedagógicas y técnicas), } \\
\text { a fin de orientar a los alumnos en } \\
\text { la construcción de su aprendizaje } \\
\text { significativo y, fundamentalmente, } \\
\text { propiciar que los docentes se conviertan } \\
\text { en el factor clave para la creación de } \\
\text { ambientes educativos colaborativos } \\
\text { hacia la generación y aplicación del } \\
\text { conocimiento con colegas y estudiantes }\end{array}$ \\
\hline
\end{tabular}




\begin{tabular}{|c|c|c|c|c|}
\hline Año & Autores & Título & $\begin{array}{c}\text { Categoría Central } \\
\text { de Análisis }\end{array}$ & Postura Argumentativa \\
\hline 2013 & $\begin{array}{l}\text { Maria del } \\
\text { Carmen } \\
\text { Ricoy } \\
\text { Lorenzo y } \\
\text { Jennifer } \\
\text { Fernández } \\
\text { Rodríguez }\end{array}$ & $\begin{array}{l}\text { Contribuciones } \\
\text { y controversias } \\
\text { que genera el } \\
\text { uso de las TIC } \\
\text { en la educación } \\
\text { superior: un } \\
\text { estudio de caso }\end{array}$ & $\begin{array}{l}\text { TIC } \\
\text { Aprendizaje y } \\
\text { Enseñanza }\end{array}$ & $\begin{array}{l}\text { La integración de las TIC en el } \\
\text { proceso de enseñanza y aprendizaje } \\
\text { conlleva un reto substancial } \\
\text { para asumir tanto por el docente } \\
\text { como por el estudiante. Se busca } \\
\text { indagar sobre las aportaciones } \\
\text { y dificultades que produce el } \\
\text { uso de las Tecnologías en el } \\
\text { estudiante enfocado en el ámbito } \\
\text { cualitativo. Como resultados y } \\
\text { conclusiones cabe destacar que el } \\
\text { grupo estudiado, a través de la } \\
\text { reflexión y el análisis que realiza } \\
\text { en sus diarios sobre el proceso de } \\
\text { aprendizaje y el trabajo que aborda } \\
\text { con las TIC, es consciente de las } \\
\text { múltiples aportaciones de estas } \\
\text { herramientas descubriendo, además, } \\
\text { los principales obstáculos. Los } \\
\text { participantes asumen como principal } \\
\text { contribución del uso de las TIC la } \\
\text { adquisición de nuevos conocimientos } \\
\text { técnicos. Las dificultades que } \\
\text { se encuentran con la utilización } \\
\text { de las TIC son asociados con las } \\
\text { problemáticas que experimentan } \\
\text { durante el proceso de aprendizaje y } \\
\text { al deficiente funcionamiento de los } \\
\text { aparatos informáticos }\end{array}$ \\
\hline 2008 & $\begin{array}{l}\text { Julio César } \\
\text { González } \\
\text { Mariño }\end{array}$ & $\begin{array}{l}\text { TIC y la } \\
\text { transformación } \\
\text { de la práctica } \\
\text { educativa en el } \\
\text { contexto } \\
\text { de las sociedades } \\
\text { del conocimiento }\end{array}$ & $\begin{array}{l}\text { TIC } \\
\text { Enseñanza } \\
\text { Educación Superior }\end{array}$ & $\begin{array}{l}\text { La incorporación de las TIC ha jugado } \\
\text { un papel importante y ha generado } \\
\text { múltiples beneficios en muchos sectores } \\
\text { de la economía. Es importante tener } \\
\text { en cuenta que no solo la tecnología de } \\
\text { información es el agente transformador } \\
\text { de la sociedad, sino que también lo es el } \\
\text { conocimiento que es posible generar con } \\
\text { la información. } \\
\text { Es fundamental que en la enseñanza } \\
\text { en la Educación Superior, el docente } \\
\text { adquiera ciertas habilidades, } \\
\text { conocimientos y actitudes que lo } \\
\text { capaciten para aplicar estrategias } \\
\text { innovadoras y modelos alternos, que } \\
\text { incluyan la enseñanza por medio de } \\
\text { TIC, donde el profesional tenga un rol } \\
\text { activo y mayor responsabilidad de su } \\
\text { aprendizaje en el proceso }\end{array}$ \\
\hline
\end{tabular}




\begin{tabular}{|c|c|c|c|c|}
\hline Año & Autores & Título & $\begin{array}{c}\text { Categoría Central } \\
\text { de Análisis }\end{array}$ & Postura Argumentativa \\
\hline 2007 & $\begin{array}{l}\text { César Coll } \\
\text { Salvador, } \\
\text { María José } \\
\text { Rochera } \\
\text { Villach, } \\
\text { Rosa María } \\
\text { Mayordomo } \\
\text { Saíz y Mila } \\
\text { Naranjo } \\
\text { Llanos }\end{array}$ & $\begin{array}{l}\text { Evaluación } \\
\text { continua y ayuda } \\
\text { al aprendizaje. } \\
\text { Análisis de una } \\
\text { experiencia de } \\
\text { innovación en } \\
\text { educación } \\
\text { superior con } \\
\text { apoyo de las TIC }\end{array}$ & $\begin{array}{l}\text { TIC } \\
\text { Educación Superior } \\
\text { Sistema de } \\
\text { Evaluación de } \\
\text { Aprendizaje }\end{array}$ & $\begin{array}{l}\text { Se incorpora un sistema integrado } \\
\text { de evaluación continua (SIEC) } \\
\text { en la educación superior para } \\
\text { dar a obtener evidencias en los } \\
\text { conocimientos y habilidades de los } \\
\text { estudiantes y facilitarles el proceso } \\
\text { de aprendizaje. Con el apoyo de } \\
\text { las TIC, se puede mostrar con } \\
\text { claridad resultados en el sistema } \\
\text { de evaluación del aprendizaje } \\
\text { empleados a los estudiantes de } \\
\text { Educación Superior, dándole } \\
\text { reflexión y conllevando a finalidades } \\
\text { formadoras sobre el proceso de } \\
\text { aprendizaje, el estudiante se sitúa } \\
\text { en contextos reales que permiten } \\
\text { relacionar la teoría y práctica } \\
\text { utilizado de manera contextualizada los } \\
\text { conocimientos adquiridos }\end{array}$ \\
\hline
\end{tabular}

Fuente: Elaboración de los autores.

La implementación de las TIC ha tomado un alto grado de importancia en la educación, a tal magnitud que el gobierno en conjunto con diferentes entes ha emprendido proyectos que incluyan en las aulas de clases las herramientas necesarias para que el docente pueda impartir sus clases en un aprendizaje innovador. Éstos resultados nos permiten identificar que actualmente existe una imagen positiva en los docentes sobre el papel el rol que juega las TIC en los procesos educativos, teniendo en cuenta que existen brechas entre las instituciones que no cuenten con la infraestructura suficiente para la inclusión de éstos saberes tecnológicos. Es así como el Ministerios de Educación Nacional en Colombia propende por fortalecer el proceso de formación y el sistema educativo desde las TIC.
Determinar la pertinencia de las tecnologías para el desarrollo por competencias asociadas a los perfiles profesionales juega un papel fundamental en las IES, donde las competencias genéricas y específicas de los estudiantes, egresados, académicos y empresarios, son parte esencial de la formación integral de los estudiantes, si bien, hoy día se torna muy difícil encontrar Instituciones de Educación Superior que no apunten a la incorporación de herramientas tecnológicas en el proceso de enseñanzaaprendizaje, si existen casos en donde los profesores enfrentan un gran reto para implementar las TIC en su práctica pedagógica, por lo tanto deberán rediseñarse las funciones de su quehacer educativo. En la tabla 3, se detallan los aportes más significativos sobre las competencias de desempeño implementadas en la formación profesional. 
TABla 3

Análisis sobre las competencias en la formación profesional

\begin{tabular}{|c|c|c|c|c|}
\hline Año & Autores & Título & $\begin{array}{c}\text { Categoría Central } \\
\text { de Análisis }\end{array}$ & $\begin{array}{c}\text { Postura } \\
\text { Argumentativa }\end{array}$ \\
\hline 2016 & $\begin{array}{l}\text { Ana Patricia } \\
\text { León } \\
\text { Urquijo, } \\
\text { Eduardo } \\
\text { Risco del } \\
\text { Valle y } \\
\text { Cristina } \\
\text { Alarcón } \\
\text { Salvo }\end{array}$ & $\begin{array}{l}\text { Estrategias } \\
\text { de aprendizaje } \\
\text { en educación } \\
\text { superior en } \\
\text { un modelo } \\
\text { curricular por } \\
\text { competencias }\end{array}$ & $\begin{array}{l}\text { Aprendizaje } \\
\text { Educación Superior } \\
\text { Competencias }\end{array}$ & $\begin{array}{l}\text { Los temas que se estudian } \\
\text { como el internet, los libros y las } \\
\text { preguntas al profesor favorecen la } \\
\text { autorregulación del aprendizaje; sin } \\
\text { embargo, no todos los estudiantes } \\
\text { utilizan las estrategias de } \\
\text { evaluación como revisar el orden } \\
\text { de los temas a analizar, decidir } \\
\text { cuándo terminar de hacerlo, } \\
\text { realizar pausas mientras estudian } \\
\text { para descansar, y en el momento, } \\
\text { algunos no siempre están atentos } \\
\text { al tema de estudio, ni mantienen } \\
\text { la motivación y la concentración, } \\
\text { así como les faltan voluntad y } \\
\text { decisión que algunas veces generan } \\
\text { angustia. Por lo tanto, es necesario } \\
\text { que aprendan a organizar el tiempo, } \\
\text { de tal forma que puedan dedicarlo } \\
\text { a sus diferentes actividades de } \\
\text { formación profesional, de vida } \\
\text { familiar, social y laboral. }\end{array}$ \\
\hline 2015 & $\begin{array}{l}\text { Jaime } \\
\text { Castrillón, } \\
\text { Leonor } \\
\text { Cabeza } \\
\text { y Jahir } \\
\text { Lombana }\end{array}$ & $\begin{array}{l}\text { Competencias } \\
\text { más } \\
\text { importantes } \\
\text { para la } \\
\text { disciplina } \\
\text { administrativa } \\
\text { en Colombia }\end{array}$ & $\begin{array}{l}\text { Competencias } \\
\text { Genéricas y } \\
\text { Específicas } \\
\text { Conocimiento } \\
\text { Educación Superior }\end{array}$ & $\begin{array}{l}\text { La percepción sobre la generación } \\
\text { de competencias genéricas y } \\
\text { específicas más importantes para } \\
\text { los Administradores en Colombia, } \\
\text { reflejan diferentes percepciones } \\
\text { sobre académicos y empleadores. } \\
\text { En el caso de los académicos, } \\
\text { consideran que los administradores } \\
\text { están orientados a los elementos } \\
\text { epistemológicos, tanto en las } \\
\text { competencias genéricas como } \\
\text { específicas. Para los empleadores } \\
\text { es más importante el trabajo en } \\
\text { equipo que aplicar el conocimiento } \\
\text { a la práctica; por lo tanto, para la } \\
\text { aplicación de sus conocimientos, la } \\
\text { empresa proporcionará procesos } \\
\text { de inducción al trabajador cuando } \\
\text { inicie su vida laboral. } \\
\text { Finalmente, es necesario hacer } \\
\text { un esfuerzo para cerrar la brecha } \\
\text { entre los conocimientos y los } \\
\text { aspectos prácticos frente a los } \\
\text { conceptos éticos, morales y los } \\
\text { principios ontológicos mediante una } \\
\text { buena educación impartida en las } \\
\text { instituciones de educación superior }\end{array}$ \\
\hline
\end{tabular}




\begin{tabular}{|c|c|c|c|c|}
\hline Año & Autores & Título & $\begin{array}{c}\text { Categoría Central } \\
\text { de Análisis }\end{array}$ & $\begin{array}{c}\text { Postura } \\
\text { Argumentativa }\end{array}$ \\
\hline 2014 & $\begin{array}{l}\text { Johann } \\
\text { Pirela } \\
\text { Morillo y } \\
\text { José de Jesús } \\
\text { Cortés Vera }\end{array}$ & $\begin{array}{l}\text { El desarrollo de } \\
\text { competencias } \\
\text { informacionales } \\
\text { en estudiantes } \\
\text { universitarios. } \\
\text { Experiencia y } \\
\text { perspectivas } \\
\text { en dos } \\
\text { universidades } \\
\text { latino- } \\
\text { americanas }\end{array}$ & $\begin{array}{l}\text { Competencias } \\
\text { Informacionales } \\
\text { Formación } \\
\text { Profesional } \\
\text { Instituciones } \\
\text { Educativas }\end{array}$ & $\begin{array}{l}\text { Avanzar en una mejor comprensión } \\
\text { para plantear estrategias } \\
\text { institucionales en materia de } \\
\text { desarrollo de competencias } \\
\text { informacionales, puede llegar } \\
\text { a considerarse en un gran reto } \\
\text { para las instituciones educativas; } \\
\text { considerando la incorporación de } \\
\text { la sociedad a las dinámicas del } \\
\text { conocimiento. Es allí, como las } \\
\text { Instituciones de Educación Superior } \\
\text { deben garantizar la formación de } \\
\text { profesionales críticos y reflexivos } \\
\text { que aprendan a agregarle valor a } \\
\text { la información con efectividad y de } \\
\text { esta forma lograr el desarrollo de } \\
\text { competencias para la apropiación } \\
\text { crítica de la información, como base } \\
\text { del aprendizaje a lo largo de toda } \\
\text { su vida }\end{array}$ \\
\hline 2013 & $\begin{array}{l}\text { Jordi } \\
\text { Planas-Coll }\end{array}$ & $\begin{array}{l}\text { El } \\
\text { contrasentido } \\
\text { de la enseñanza } \\
\text { basada en } \\
\text { competencias. }\end{array}$ & $\begin{array}{l}\text { Enseñanza Basado } \\
\text { en Competencias } \\
\text { Instituciones de } \\
\text { Educación Superior }\end{array}$ & $\begin{array}{l}\text { En la asociación de la } \\
\text { Enseñanza Basado en } \\
\text { Competencias (EBC) en la } \\
\text { educación formal, se presenta } \\
\text { confusión en las competencias } \\
\text { reales de las personas que } \\
\text { interactúan en los ciclos } \\
\text { educativos. La estandarización } \\
\text { de manera artificial de la EBC, } \\
\text { genera una desinformación } \\
\text { en el mercado de trabajo y } \\
\text { en el sistema educativo, esto } \\
\text { debido a la escasa atención } \\
\text { presentada en los ciclos } \\
\text { asociados a la enseñanza basada } \\
\text { en competencias realizadas en } \\
\text { las Instituciones de Educación } \\
\text { Superior }\end{array}$ \\
\hline
\end{tabular}

Fuente: Elaboración de los autores.

En éste escenario, es deseable para las Instituciones de Educación Superior garantizar la formación de los profesionales con un alto sentido crítico y reflexivo, que permitan plantear soluciones y alternativas a los posibles problemas e inconvenientes presentados en el entorno. Lograr el desarrollo de competencias de desempeño, aportará a la efectividad y apropiación de la información como base para el aprendizaje. Para ello, debe existir una acción mancomunada entre todos los actores de la comunidad educativa con las normativas establecidas por las entidades gubernamentales. 
Igualmente, las políticas y lineamientos institucionales de orden académico, deben estipularse con el objeto de fortalecer el desarrollo de competencias de desempeño que contribuyan la producción, transferencia del conocimiento y la tecnología. Para que exista un mejoramiento continuo entre la enseñanza y el aprendizaje deben diseñarse contenidos programáticos innovadores, desarrollando en el estudiante sus capacidades para aprender a aprender, es decir, lograr aplicar los procesos de pensamiento a la comprensión lectora y construcción del conocimiento, direccionándolos a la resolución de problemas y fortalecimiento de sus desarrollo intelectual.

\section{Discusión y CoNCLUSIONES}

A partir de la revisión sistemática se logró inferir cómo la implementación de herramientas informáticas en las Instituciones de Educación Superior aportan significativamente al desarrollo y promoción de las competencias de desempeño, orientadas a implementar nuevas alternativas de innovación educativa, que propicien escenarios de aprendizaje colaborativo y significativos en la mediación docente-estudiante. Se valida así la evolución progresiva de las competencias que han ayudado a generar interacción entre la academia y la sociedad, enmarcadas acorde a los estándares de calidad en la educación.

En el análisis de las categorías teóricas: calidad educativa, competencias de desempeño y aplicación de las tecnologías, emergen relaciones explícitas o subyacentes consentido de interdependencia entre sí. Además, se perciben complejidades estructurales y funcionales en sus procesos relacionales desde una visión sistémica, donde las categorías hacen importantes aportes a la comprensión del objeto y sus interacciones.

Las tendencias en el uso de las TIC para el desarrollo de las competencias de desempeño en la Educación Superior, abre un horizonte de investigación e innovación que a su vez formula nuevos retos de transformación curricular, orientados al desarrollo de los estándares nacionales y los referentes internacionales. Finalmente, la dinámica descrita en el presente artículo es un espacio susceptible de ser validado a partir de la visión crítica de otros investigadores, como un aporte significativo a la generación de nuevos objetos de estudio.

\section{REFERENCIAS}

Altbach, P. \& Knight, J. (2006). Visión panorámica de la internacionalización en la educación superior: motivaciones y realidades. Perfiles educativos, 28(112). 13-39.

Alvarado, E., Morales, D. y Aguayo, E. (2016). Percepción de la calidad educativa: caso aplicado a estudiantes de la Universidad Autónoma de Nuevo León y del Instituto Tecnológico de Estudios Superiores de Monterrey. Revista de la educación superior, 45(180). 55-74.

Barbón, O. y Fernández, J. (2018). Rol de la gestión educativa estratégica en la gestión del conocimiento, la ciencia, la tecnología y la innovación en la educación superior. Educación Médica, 19(1), 51-55.

Castrillón, J., Cabeza, L. y Lombana, J. (2015). Competencias más importantes para la disciplina administrativa en Colombia. Contaduría y administración, 60(4), 776-795. http://dx.doi.org/10.1016/j. cya.2015.07.009 
Coll, C., Rochera, M., Mayordomo, R. y Naranjo, M. (2007). Evaluación continua y ayuda al aprendizaje. Análisis de una experiencia de innovación en educación superior con apoyo de las TIC. Electronic Journal of Research in Educational Psychology, 5(3), 783-804.

Espinoza, O. y González, L. (2012). Estado actual del sistema de aseguramiento de la calidad y el régimen de acreditación en la educación superior en Chile. Revista de la educación superior, 41(162), 87-109.

Fernández, S. y Ruzo, E. (2004). Los procesos de internacionalización y globalización en la educación superior: un análisis de los países de la OCDE. Revista de educación, 335(1). 385-413.

Karam, J., Buitrago, A., Fagua, A. y Romero, Y. (2013). Estrategias de gamificación aplicadas al diagnóstico de la incorporación pedagógica de las TIC en una comunidad académica. Cultura, Educación y Sociedad, 4(1). 55-74.

León, A., Risco, E. y Alarcón, C. (2014). Estrategias de aprendizaje en educación superior en un modelo curricular por competencias. Revista de la educación superior, 43(172). 123-144.

Marín, F., Inciarte, A., Hernández, H. y Pitre, R. (2017). Estrategias de las Instituciones de Educación Superior para la Integración de las Tecnología de la Información y la Comunicación y de la Innovación en los Procesos de Enseñanza. Un Estudio en el Distrito de Barranquilla, Colombia. Formación universitaria, 10(6), 2938. http://dx.doi.org/10.4067/S071850062017000600004

Marín-González, F., Cabas, L., Cabas, L. y Paredes-Chacín, A. (2018). Formación Integral en Profesionales de la Ingeniería. Análisis en el Plano de la Calidad Educativa. Formación universitaria, 11(1), 13-24. http://dx.doi.org/10.4067/ S0718-50062018000100013
González, J. (2008). TIC y la transformación de la práctica educativa en el contexto de las sociedades del conocimiento. RUSC. Universities and Knowledge Society Journal, 5(2), 1-8. Moher, D., Liberati, A., Tetzlaff, J., Altman, D. \& The Prisma Group. (2009). Preferred reporting ítems for systematic reviews and meta-analyses: The PRISMA statement. Plos One. 6(7). 1-8. https://doi.org/10.1371/ journal.pmed.1000097

Mollis, M. (2014). Administrar la crisis de la educación pública y evaluar la calidad universitaria en América Latina: dos caras de la misma reforma educativa. Revista de la educación superior, 43(169). 25-45. https://doi. org/10.1016/j.resu.2014.01.001

Moreno, C. (2017). Las reformas en la educación superior pública en México: rupturas y continuidades. Revista de la educación superior, 46(182). 27-44.

Nolasco, P. y Ojeda, M. (2016). La Evaluación de la Integración de las TIC en la Educación Superior: Fundamento para una Metodología. RED-Revista de Educación a Distancia, 9(48). $1-24$.

Orozco, L. (2010). Calidad académica y relevancia social de la educación superior en América Latina. Revista iberoamericana de educación superior, 1(1). 24-36.

Planas-Coll, J. (2013). El contrasentido de la enseñanza basada en competencias. Revista iberoamericana de educación superior, 4(10), 75-92. https://doi. org/10.1016/S2007-2872(13)71925-5

Pedraza, N., Farías, G., Lavín, J. y Torres, A. (2013). Las competencias docentes en TIC en las áreas de negocios y contaduría Un estudio exploratorio en la educación superior. Perfiles educativos, 35(139), 8-24. 
Pinto, A. y Díaz, J. (2015). Convivencia Escolar en la era de la hiperconectividad. Cultura Educación y Sociedad, 6(1). 149-164.

Pirela, J. y Cortés, J. (2014). El desarrollo de competencias informacionales en estudiantes universitarios: Experiencia y perspectivas en dos universidades latinoamericanas. $I n$ vestigación bibliotecológica, 28(64), 145-172. https://doi.org/10.1016/ S0187-358X(14)70913-X

República de Colombia. (8 de febrero de 1994). Por el cual se expide la ley general de educación. [Ley 115]. DO: 41.214 .

República de Colombia. Congreso de la República. (28 diciembre de 1992). Por el cual se organiza el servicio público de la Educación Superior. [Ley 30]. DO: 40700.

República de Colombia. Ministerio de Educación Nacional (2013). Competencias TIC para el desarrollo profesional docente. Bogotá, D.C.: Mineducación.

Ricoy, M. y Fernández, J. (2013). Contribuciones y controversias que genera el uso de las TIC en la educación superior: un estudio de caso. Revista de Educación, 360.

Sunkel, G., Trucco, D. y Espejo, A. (2014). La Integración de las Tecnologías Digitales en las Escuelas de América Latina y el Caribe. Santiago de Chile: CEPAL. Recuperado de https://goo.gl/gVoZDr

Tobón, S. (2006). Las Competencias en la Educación Superior. Políticas de Calidad. Bogotá, D.C.: ECOE.

UNESCO. (2008). Normas sobre competencias en TIC para Docentes. París: UNESCO.
Carolina Mercado-Porras es profesional en Finanzas y Relaciones Internacionales, Gestor de Investigación adscrita a la Vicerrectoría de Investigación \& Desarrollo, vinculada al Grupo de Investigación en Economia y Finanzas (FPI), Universidad de la Costa.

Yuneidis Morales-Ortega es Especialista en Gerencia Financiera, Profesional en Finanzas y Relaciones Internacionales, adscrita al Departamento de Planeación, vinculada al grupo de investigación Gicade, Universidad de la Costa. 